\title{
BMJ Open Cohort profile: the mature adults cohort of the Malawi longitudinal study of families and health (MLSFH-MAC)
}

\author{
Iliana V Kohler (10 , ${ }^{1,2}$ Chiwoza Bandawe, ${ }^{3}$ Alberto Ciancio, ${ }^{2,4}$ Fabrice Kämpfen, ${ }^{2}$ \\ Collin F Payne, ${ }^{5}$ James Mwera, ${ }^{6}$ James Mkandawire, ${ }^{6}$ Hans-Peter Kohler ${ }^{1,2}$
}

To cite: Kohler IV, Bandawe C, Ciancio A, et al. Cohort profile: the mature adults cohort of the Malawi longitudinal study of families and health (MLSFH-MAC). BMJ Open 2020;10:e038232. doi:10.1136/ bmjopen-2020-038232

- Prepublication history and additional material for this paper are available online. To view these files, please visit the journal online (http://dx.doi. org/10.1136/bmjopen-2020038232).

Received 05 March 2020 Revised 18 July 2020

Accepted 19 August 2020

Check for updates

(C) Author(s) (or their employer(s)) 2020. Re-use permitted under CC BY-NC. No commercial re-use. See rights and permissions. Published by BMJ.

${ }^{1}$ Sociology, University of Pennsylvania, Philadelphia, Pennsylvania, USA

${ }^{2}$ Population Studies Center, University of Pennsylvania, Philadelphia, Pennsylvania, USA ${ }^{3}$ Department of Mental Health, University of Malawi, College of Medicine, Blantyre, Malawi ${ }^{4}$ Department of Economics, University of Lausanne,

Lausanne, Switzerland

${ }^{5}$ School of Demography, Research School of Social Sciences, Australian National University, Canberra, New South Wales, Australia

${ }^{6}$ Invest in Knowledge Initiative (IKI), Zomba, Malawi

Correspondence to

Dr lliana V Kohler;

iliana@upenn.edu

\section{ABSTRACT}

Purpose The Mature Adults Cohort of the Malawi Longitudinal Study of Families and Health (MLSFH-MAC) contributes to global ageing studies by providing a rare opportunity to study the processes of individual and population ageing, the public health and social challenges associated with ageing and the coincident shifts in disease burdens, in a low-income, high HIV prevalence, subSaharan African (SSA) context.

Participants The MLSFH-MAC is an open populationbased cohort study of mature adults aged $45+$ years living in rural communities in three districts in Malawi. Enrolment at baseline is 1266 individuals in 2012. Follow-ups were in 2013, 2017 and 2018 when the cohort size reached 1626 participants in 2018.

Findings to date Survey instruments cover ageingrelated topics such as cognitive and mental health, noncommunicable diseases (NCDs) and related health literacy, subjective survival expectations, measured biomarkers including HIV, grip strength, hypertension, fasting glucose, body mass index (BMI), broad individual-level and household-level social and economic information, a 2018 qualitative survey of mature adults and community officials, 2019 surveys of village heads, healthcare facilities and healthcare providers in the MLSFH-MAC study areas. Across many domains, MLSFH-MAC allows for comparative research with global ageing studies through harmonised measures and instruments. Key findings to date include a high prevalence of depression and anxiety among older adults, evidence for rapid declines in cognitive health with age, a low incidence of HIV among mature adults, rising prevalence of HIV due to increased survival of HIV-positive individuals and poor physical health with high NCD prevalence.

Future plans An additional wave of MLSFH-MAC is forthcoming in 2021, and future expansions of the cohort are planned. MLSFH-MAC data will also be publicly released and will provide a wealth of information unprecedented for ageing studies in a low-income SSA context that broadly represents the socioeconomic environment of millions of individuals in south-eastern Africa.

\section{INTRODUCTION}

Mature adults (=individuals aged 45+ years old) constitute a demographically, economically and socially important subset of societies

\section{Strengths and limitations of this study}

Mature Adults Cohort of the Malawi Longitudinal Study of Families and Health (MLSFH-MAC) is a longitudinal global ageing study in a rural low-income context that provides up to 20 years of data on the life course of cohort members.

- MLSFH-MAC broadly represents the socioeconomic environment of millions of individuals in south- eastern Africa, and it provides a wealth of information that is rare for ageing studies in a low-income subSaharan African (SSA) context.

- MLSFH-MAC is at the vanguard in measuring longitudinal changes in cognitive and mental health among older individuals in SSA.

- MLSFH-MAC allows comparative research with global ageing studies through harmonised measures and instruments.

- MLSFH-MAC is not a nationally representative sample, but broadly represents mature adults in rural Malawi where $85 \%$ of the population lives.

in sub-Saharan African (SSA) countries. Yet, due to a relative lack of ageing studies in SSA or low-income countries (LICs), very little is known about the health and socioeconomic contexts of older individuals in SSA LICs. Long-term cohort studies covering older adults, which are critical for understanding life-course determinants of health and ageing, are particularly rare. To fill this niche, the "Mature Adults Cohort of the Malawi Longitudinal Study of Families and Health (MLSFH-MAC)' was initiated in 2012. It is conducted in one of the world's poorest countries, where a per-capita GDP (gross domestic product) is equal to about $4 \%$ of the global average, and is a collaboration between the University of Pennsylvania, the College of Medicine and Invest in Knowledge Initiative (IKI), both in Malawi. The key innovation of the MLSFH-MAC is that it provides a rare opportunity to better understand the processes of individual and population ageing, the public health and social 
challenges associated with ageing and the coincident shifts in disease burdens, in a low-income SSA context where very few comparable ageing studies exist. The cohort has been followed up in 2013, 2017 and 2018, with a planned data collection in 2021.

The contribution of the MLSFH-MAC to global ageing studies is important as patterns of ageing and disease trajectories, as well as the appropriate policy and healthsystems responses, are distinctly different in SSA LICs from those that have been extensively studied in higher income populations. Few countries in the region have developed effective and/or viable health policies and responses to address the critical confluence of ageing, with its profound social and economic implications, and shifting disease burdens towards non-communicable diseases (NCDs). ${ }^{1}$ Analyses of the MLSFH-MAC can help inform social policies and health sector strategies that are required for the growing population of older adults in SSA countries.

\section{COHORT DESCRIPTION}

\section{Study population}

The MLSFH-MAC is an ongoing open population-based cohort study of mature adults aged 45 years and older. Enrolment is 1266 in 2012, 1257 in 2013, 1606 in 2017 and 1626 in 2018 when last surveyed. The cohort is set in rural communities in three districts in Malawi: Balaka in the southern region, Mchinji in the central region and Rumphi in the northern region. A unique aspect of the MLSFH-MAC is that it was derived from the Malawi Longitudinal Study of Families and Health (MLSFH), an ongoing longitudinal panel study established in 1998 that examines how families and individuals cope with the social, economic, demographic and health consequences of the HIV/AIDS epidemic. ${ }^{2}$ The MLSFH-MAC cohort was established by selecting MLSFH respondents aged 45+ years in 2012, and enrolling them in an extensive ageing and health baseline survey in 2012 . MLSFH-MAC data can be linked to the prior MLSFH lifecourse and contextual information dating back to 1998. In combination, therefore, the MLSFH-MAC provides two decades of longitudinal cohort data across mid to older ages, thereby providing exceptional insights into how individual and population ageing has unfolded across 20 years in a poor rural context with high HIV prevalence that broadly represents the socioeconomic environment of millions of individuals in south-eastern Africa.

The key inclusion criteria in 2012 for enrolment in the MLSFH-MAC were twofold: (1) being a MLSFH respondent aged 45 years or older in 2012; and (2) having been interviewed in both the 2008 and 2010 MLSFH data collection rounds. The second criteria ensured that at least three waves of mental health and subjective well-being data were available for each baseline participant in 2012. Baseline enrolment in the MLSFH-MAC included 1266 individuals clustered in $130+$ villages, representing more than $90 \%$ of the 1402 eligible MLSFH respondents who met the enrolment criteria (=target sample) (figure 1). Migration out of the study areas and mortality were the primary reasons for not enrolling eligible respondents. At each follow-up, the initial study population was augmented with additional MLSFH respondents who reached eligibility. To ensure an adequate representation of HIV-positive (HIV+) individuals in the cohort, age-eligible HIV+ respondents were enrolled if they participated in either the 2008 or 2010 MLSFH data collection. Summary statistics for the 2018 MLSFH-MAC cohort are reported in table 1, and comparisons of the study population with nationally representative samples are reported in the online supplemental materials.

\section{MLSFH-MAC data collections}

Figure 1 describes enrolment in, and attrition from the MLSFH-MAC cohort since 2012. Additional analyses show that the MLSFH-MAC study baseline population closely matches the rural subsample in the 2010 nationally representative Integrated Household Survey (IHS3) in key observable characteristics (online supplemental materials). At each follow-up, all existing MLSFHMAC-eligible respondents were re-interviewed (or approached for interview), and newly-eligible MLSFH respondents were added to the MLSFH-MAC cohort. The largest number of respondents was added in 2017 (423 new respondents), while few respondents newly reached eligibility in 2013 and 2018 (12 and 56, respectively). As a result of this open cohort design, some MLSFH-MAC cohort members have participated in fewer study follow-ups. In 2017 and 2018, the MLSFH-MAC conducted extensive migration follow-ups of respondents who moved to other areas within Malawi. Eligible respondents who were not successfully interviewed in a given year, generally because they were temporarily absent during three contact attempts, were continued to be approached for interview in any follow-up data collections. Table 2 reports reasons for attrition between the 2012 baseline survey and the (currently) last follow-up in 2018. Supplemental analyses show that the initial enrolment is not selective, and that attrition is positively related to several baseline (2012) characteristics such as being male, being older, having no formal schooling, not being married in 2012, being poor, scoring low on the indicators of subjective well-being, mental or physical health and being depressed (online supplemental materials). Of the 181 respondents lost to follow-up during 2012 to 2018, the majority (149) died, and many of the characteristics predicting attrition are predictors of mortality as the most important reason for attrition in the MLSFH-MAC cohort during 2012 to 2018. Overall, of 1266 respondents interviewed at baseline, $86 \%$ were successfully found in 2018. Excluding deceased respondents, the MLSFH-MAC successfully surveyed in 2018 a remarkable $97 \%$ of the respondents 


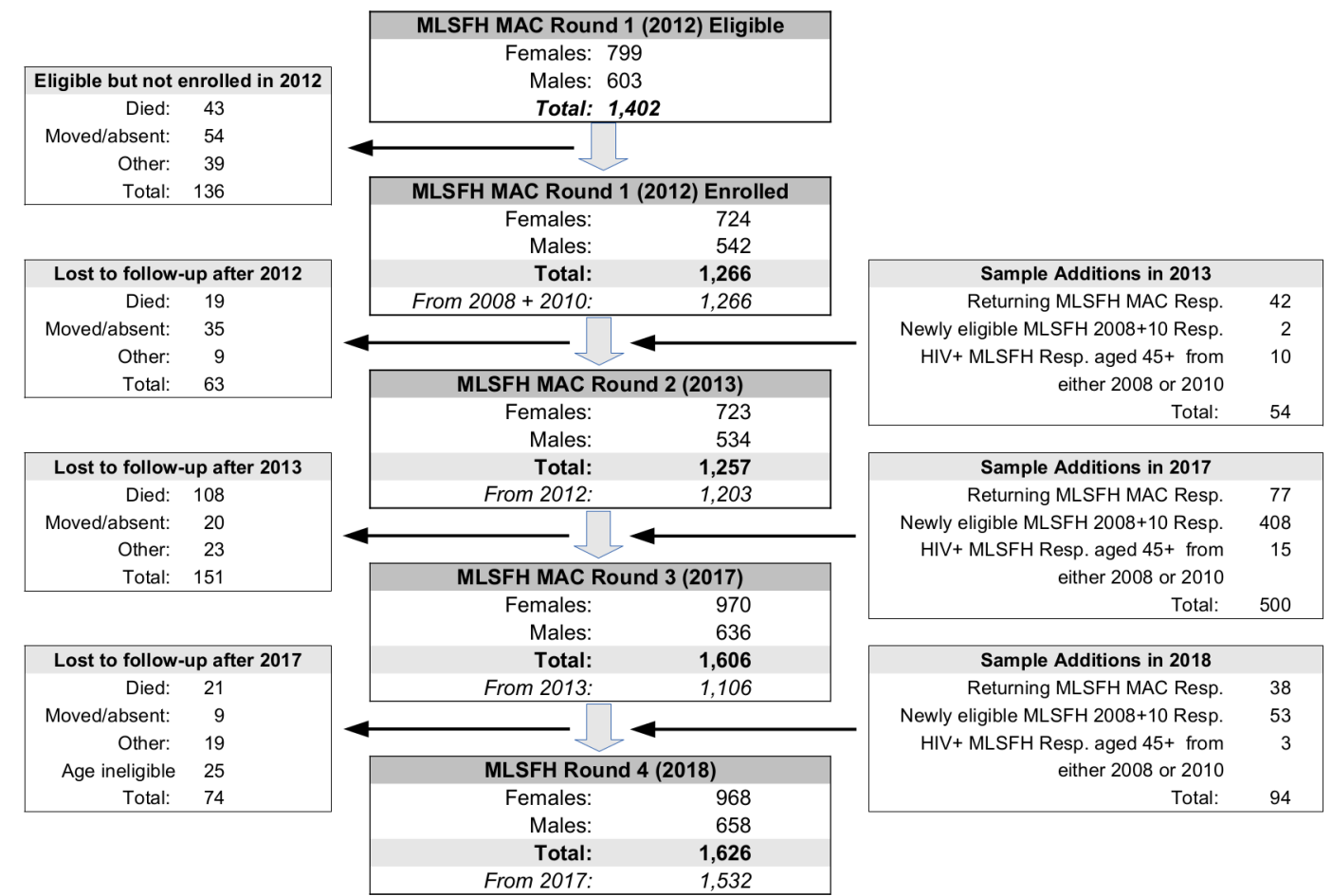

Figure 1 Initial MLSFH-MAC sample selection, additional enrolments, mortality and sample attrition during 2012 to 2018 Notes: MLSFH-MAC is based in three districts in Malawi: Rumphi in the north, Mchinji in the centre, and Balaka in the South. MLSFH-MAC sampling and related relevant data collection procedures are described in the online supplemental materials. 'Returning MLSFH-MAC Respondents' refers to respondents who were interviewed in the current MLSFH-MAC wave, and were eligible but not successfully surveyed in the prior MLSFH-MAC wave (usually because they were temporarily absent). MLSFHMAC study instruments are summarised in table 3. HIV+, HIV-positive; MLSFH-MAC, Mature Adults Cohort of the Malawi Longitudinal Study of Families and Health; Resp., respondents.

interviewed at baseline, providing a very high rate of retention of study participants in this cohort.

\section{MLSFH-MAC measurements}

Surveys have been conducted in the local languages (Chichewa, Chiyao and Chitumbuka) by experienced interviewers and/or HIV testing counsellors who are extensively trained in the study methodology and instruments at each data collection round. The survey instruments (table 3) are a combination of previously-existing MLSFH instruments and newly developed survey instruments covering in particular ageing-related topics such as cognitive and mental health, NCDs, NCD-related health literacy and so on. This survey design offers the advantage of allowing longitudinal comparability of cohort data since 1998, thereby facilitating life-course studies (table 4), while also allowing for an expansion of the survey into new ageing and NCD-related dimensions for which prior data do not exist (table 3). New survey modules (ie, mental/cognitive health instruments) were extensively pretested during focus-group interviews and pilot tests. In most cases, newly implemented instruments were also harmonised as much as possible with other ageing studies (such as the Health and Retirement Survey and its sister-studies) that provide wide-ranging multidisciplinary data on ageing in high-income and middle-income countries. ${ }^{3}$ The study also includes extensive information that complements the individual-level data in table 3 , including a 2017 study focussed on pessimistic survival expectations, ${ }^{4}$ a 2018 qualitative study of mature adults and community officials and a 2019 survey information from village heads, healthcare facilities and healthcare providers in the MLSFH-MAC study areas (see online supplemental materials for further details).

\section{Patient and public involvement}

The MLSFH-MAC includes eligible study participants from the general population, and does not include patients or clinic-based study populations. The MLSFH has long-standing ties to its study communities in Malawi, including through regular feedback of key study findings to the communities, local stake-holders such as village heads or village committees and local healthcare providers. Study results are also disseminated to the research community in Malawi, as well as key stake holders in the government. MLSFH-MAC study instruments were interactively developed with the study partners in Malawi, and instruments were extensively pretested, including by eliciting feedback on study design and instruments from pilot participants and participants in focus group interviews. Qualitative studies have measured the perception of the MLSFH-MAC by the study communities, and interviewers who are recruited from the local communities ensure a high acceptance of the study team by the local communities. 


\begin{tabular}{|c|c|c|c|}
\hline & Women & Men & Total \\
\hline $\begin{array}{l}\text { Number of enrolled MLSFH- } \\
\text { MAC respondents }\end{array}$ & 968 & 658 & 1626 \\
\hline \multirow[t]{2}{*}{ Age } & 59.7 & 60.6 & 60.1 \\
\hline & (11.9) & (11.6) & (11.8) \\
\hline \multicolumn{4}{|l|}{ Age group } \\
\hline$<45$ & 0.02 & 0.01 & 0.02 \\
\hline $45-54$ & 0.39 & 0.36 & 0.38 \\
\hline $55-64$ & 0.28 & 0.28 & 0.28 \\
\hline $65-74$ & 0.18 & 0.20 & 0.18 \\
\hline $75+$ & 0.13 & 0.15 & 0.14 \\
\hline \multicolumn{4}{|l|}{ Schooling attainment } \\
\hline No formal schooling & 0.37 & 0.19 & 0.29 \\
\hline Primary schooling & 0.60 & 0.70 & 0.64 \\
\hline Secondary or higher & 0.03 & 0.12 & 0.07 \\
\hline Muslim & 0.27 & 0.24 & 0.26 \\
\hline Currently married & 0.60 & 0.94 & 0.74 \\
\hline $\mathrm{HIV+}$ & 0.086 & 0.071 & 0.080 \\
\hline Female & -- & -- & 0.60 \\
\hline
\end{tabular}

Means with SDs in parentheses (where applicable). HIV status is indicated as HIV+ if any HIV test in 2006, 2008, 2012 or 2017 indicated infection with HIV.

MLSFH-MAC, Mature Adults Cohort of the Malawi Longitudinal Study of Families and Health.

\section{FINDINGS TO DATE}

\section{Mental health}

No large-scale longitudinal population-based data on depression and anxiety using clinically-validated instruments were available for SSA LICs prior to this study. Methodologically, MLSFH-MAC has demonstrated that the collection of such data is possible in a SSA LIC mature

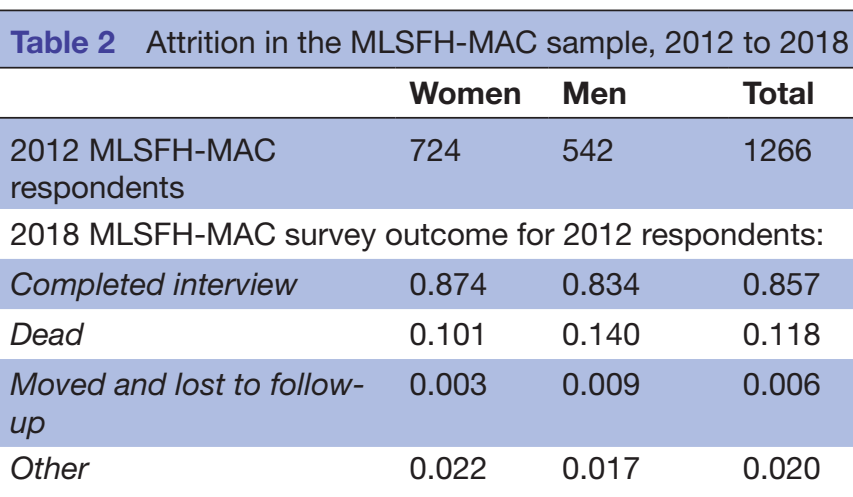

Survey outcome 'moved' includes respondents who moved and were not found during the migration follow-ups in 2017 and 2018. 'Other' includes respondents who were not successfully interviewed because of various reasons such as temporarily absent, hospitalised, and so on.

MLSFH-MAC, Mature Adults Cohort of the Malawi Longitudinal Study of Families and Health. adult population with very low awareness about mental disorders. ${ }^{5}$ Substantively, our studies found that depression and anxiety are more frequent among women than men in rural Malawi, and individuals are often affected by both. Depression and anxiety also increase substantially with age (figure 2), and mature adults can expect to spend a substantial fraction of their remaining lifetime-for instance, $52 \%$ for a 55-year-old woman-affected by poor mental health. ${ }^{6}$ The positive age gradients of depression/anxiety are not due to cohort effects, and they are in sharp contrast to high-income contexts where older individuals often experience lower levels of depression/anxiety. While socioeconomic and risk/uncertaintyrelated stressors are strongly associated with depression and anxiety, they do not explain the positive age gradients and gender gap in depression/anxiety. ${ }^{6}$ Stressors related to physical health, however, do. For men, a longer time spent outside marriage is associated with worse mental health, whereas marital dissolutions are linked to better mental health for women. ${ }^{7}$

Longitudinal analyses of the age trajectories of mental health are shown in figure 3. Importantly, the boxplots document an increase in the prevalence of depression as cohort members get older, confirming the above crosssectional pattern: in all but the youngest age group, the median PHQ-9 (9-item Patient Health Questionnaire) depression score increased as cohort members aged, and in the oldest subset of MLSFH-MAC respondents (aged $75+$ in 2018), more than $50 \%$ of the respondents experienced mild or worse depressive symptoms in 2018, an increase from only $33 \%$ in 2012, and $87 \%$ of respondents in this age group reported at least some depressive symptoms.

In summary, the above findings indicate a relatively high prevalence of poor mental health among mature adults in rural Malawi that increases strongly with age and affects women more than men. Currently, poor mental health is inadequately addressed by health systems and health policies in Malawi and most other low-income countries, and our findings highlight for the urgency of expending these services.

\section{Cognitive health}

The MLSFH-MAC is a vanguard in measuring cognitive health among older individuals in a SSA LICs. Crosssectional analyses of the 2012/2013 data found that women have substantially worse cognitive health than men, and experience a steeper age gradient in cognitive abilities. ${ }^{8}$ Strong social ties and exposure to socially complex environments were associated with higher cognitive health, as was higher life-course socioeconomic status. Poor cognitive health is associated with adverse outcomes such as less nutrition intake, lower income and reduced work efforts. Lower levels of cognitive health are also strongly associated with increased levels of depression and anxiety, and were associated with worse physical health. Cognitive health predicts mortality in the cohort until 2018, but does not predict other types of attrition. 
Table 3 Selected ageing and health-related MLSFH-MAC measurements (longitudinal data is available for most of these measures)

\begin{tabular}{|c|c|}
\hline Construct definition & Measurement/scales/items source \\
\hline Mental health and depression & $\begin{array}{l}\text { SF12 mental health score; Primary Care Evaluation of Mental Disorders (PRIME-MD), including } \\
\text { PHQ-9 depression module and GAD-7 anxiety module; subjective well-being. }\end{array}$ \\
\hline $\begin{array}{l}\text { Physical health and } \\
\text { performance }\end{array}$ & $\begin{array}{l}\text { Subjective health assessments; hand grip strength (measured using hand-held dynamometer); } \\
\text { measured height, weight and body mass index; blood pressure ( } 2013 \text { and } 2017 \text {; measured } \\
\text { using upper-arm blood pressure monitors); activities of daily living; biomarker-based HIV status } \\
\text { (2012 and 2017). }\end{array}$ \\
\hline Alcohol and tobacco use & $\begin{array}{l}\text { Alcohol use based on the Alcohol Use Disorder Identification Test (AUDIT); tobacco } \\
\text { consumption (current/ever smoked and amount). }\end{array}$ \\
\hline $\begin{array}{l}\text { Subjective risk assessments } \\
\text { and probabilistic expectations }\end{array}$ & $\begin{array}{l}\text { Interactive probabilistic expectation elicitation method developed for Malawi and low literacy } \\
\text { populations, including about mortality/survival, own HIV infection, local HIV prevalence and } \\
\text { prevalence of local AIDS-related morbidity. }\end{array}$ \\
\hline $\begin{array}{l}\text { Social, demographic and } \\
\text { economic background }\end{array}$ & $\begin{array}{l}\text { Modules repeated from MLSFH questionnaire } 2008 \text { and } 2010 \text {, including income, assets, } \\
\text { economic shocks, financial and non-financial transfers, illness/mortality of family members, } \\
\text { household composition, socioeconomic context, social and human capital. }\end{array}$ \\
\hline Work efforts and productivity & $\begin{array}{l}\text { Time devoted to different work activities and intensity of work; work efforts and work-related } \\
\text { health limitations. }\end{array}$ \\
\hline
\end{tabular}

Longitudinal data is available in the MLSFH-MAC for most of the above measures. HIV status is known for all MLSFH-MAC respondents (measured in 2017 and 2012 as part of MLSFH-MAC, 2008, 2006, 2004 as part of MLSFH). All MLSFH-MAC households are geocoded, and can be linked to health infrastructure and other spatial data (roads, schools, trading centres, markets and so on). Spouses are linked in the MLSFH-MAC, and so are children if they are included in the MLSFH. Children reported by respondents in the household/family rosters are longitudinally linked in the household rosters across waves dating back to 2004. The MLSFH-MAC survey data are complemented by extensive qualitative data with a focus on mental health collected in 2018, and in 2019 the MLSFH-MAC team interviewed village heads, healthcare providers, community health workers and representatives of all healthcare facilities in the MLSFH-MAC study areas.

GAD-7, 7-item Generalised Anxiety Disorder; MLSFH-MAC, Mature Adults Cohort of the Malawi Longitudinal Study of Families and Health; PHQ-9, 9-item Patient Health Questionnaire.

Cohort analyses also confirm the patterns of cognitive decline as individuals become older over time, which had previously been suggested—but not confirmed—based on cross-sectional 2012/2013 analyses. ${ }^{8}$

\section{HIV prevalence among mature adults}

Only 60 HIV+ mature (HIV prevalence $4.8 \%$ ) were identified at baseline in 2012 (table 5). This low HIV prevalence is the result of high mortality of HIV+ cohort members prior to antiretroviral treatment (ART) that became available in the study regions only after $2008 .{ }^{9-11}$ HIV prevalence is higher among respondents who died during 2012 to 2017 than among those that survived $(7.2 \%$ vs $4.5 \%)$, indicating that, despite better availability of ART in recent years, HIV infection continues to elevate mortality risks. At follow-up in 2017, 8.3\% of respondents tested HIV-positive, corresponding to an increase of $40 \%$ since baseline. This increase is not the result of high HIV incidence, which is very low at 0.87 new infections per 1000 person years lived (only five new infections were identified). Instead, the increase in HIV prevalence is driven by newly-enrolled respondents aged 45 to 49 years: $12.6 \%$ tested HIV+ in 2017, as compared with $6.9 \%$ in that age group in 2012, and it is these 'young' mature adults—who likely benefitted more from the roll-out of ART and the resulting mortality reductions for HIV+ individuals that are elevating the HIV prevalence in the MLSFH-MAC cohort.

\section{Physical health}

Initial findings are reported for two critical indicators for understanding ageing trajectories and NCD risks: grip strength and blood pressure. Despite being often engaged in physically demanding activities, the vast majority of the MLSFH-MAC population has a grip strength below the median of the US population aged 55 to 64 years, and more than $50 \%$ have a grip strength below the $25^{\text {th }}$ percentile of the US population (figure 4A). While grip strength is weaker for older individuals, as is the case in other populations, the longitudinal comparison of grip strength in figure $4 \mathrm{~A}$ indicates a relative stability during the period of 2012 to 2017: for ages below 75 years, the median grip strength did not decline for individuals, and a decline is only present for the oldest members of the cohort. This relative stability during a 5 -year period can in part be attributed to mortality selection, as weak grip 
Table 4 Prior MLSFH-MAC and MLSFH survey rounds available for MLSFH-MAC respondents surveyed in 2018

\begin{tabular}{|c|c|c|c|}
\hline & Women & Men & Total \\
\hline $\begin{array}{l}\text { Number of MLSFH-MAC study } \\
\text { participants in } 2018\end{array}$ & 968 & 658 & 1626 \\
\hline \multicolumn{4}{|c|}{ Proportion of 2018 MLSFH-MAC respondents with linkage to: } \\
\hline 2017 MLSFH-MAC survey data & 0.95 & 0.93 & 0.94 \\
\hline 2013 MLSFH-MAC survey data & 0.67 & 0.69 & 0.67 \\
\hline 2012 MLSFH-MAC survey data & 0.65 & 0.69 & 0.67 \\
\hline 2010 MLSFH survey data & 0.98 & 0.99 & 0.99 \\
\hline 2008 MLSFH survey data & 0.99 & 0.99 & 0.99 \\
\hline 2006 MLSFH survey data & 0.68 & 0.76 & 0.71 \\
\hline 2004 MLSFH survey data & 0.63 & 0.69 & 0.66 \\
\hline 2001 MLSFH survey data & 0.65 & 0.59 & 0.62 \\
\hline 1998 MLSFH survey data & 0.58 & 0.60 & 0.59 \\
\hline
\end{tabular}

Longitudinal information covering 20 years of their life course (ie, 1998 to 2018) is available for $59 \%$ of the $2018 \mathrm{MLSFH}$ MAC respondents, and 10 years of follow-up-covering the period 2008 to 2018 - is available for $99 \%$ of MLSFH-MAC respondents. The difference in longitudinal data linkages is explained by the fact that the MLSFH expanded its sample to older respondents in 2008, which implied that a subset of current MLSFH-MAC respondents were enrolled in 2008, with the remaining MLSFH-MAC respondents either having been part of the initial 1998 MLSFH sample or having been enrolled as part of MLSFH sample expansions during 2001 to 2006 . $^{2}$ Because the 2018 MLSFH-MAC sample includes respondents who reached age $45+$ years. Only after 2012, only about two-third of the 2018 sample was surveyed at baseline in 2012.

MLSFH-MAC, Mature Adults Cohort of the Malawi Longitudinal Study of Families and Health.

strength is associated with higher 2012 to 2017 mortality. In addition, the cross-sectional decline of grip strength with age could reflect cohort differences, with older cohorts having weaker grip strength throughout life due to worse early life determinants of physical health. ${ }^{12-14}$

Conventional risk factors for hypertension, such as obesity, lack of physical activity or exposure to western diets, are not common in the MLSFH-MAC cohort: in 2017 , only about $17 \%$ had a BMI above 25 , only $5.9 \%$ had an indication of diabetes (fasting blood glucose $>5.6 \mathrm{mmol} / \mathrm{L}$ ), and most respondents reported regular and high levels of physical activity. Despite this lack of risk factors, hypertension is widespread in the cohort (figure 4B): in 2018, 67\% of all mature adults had prehypertension (systolic blood pressure $\geq 120 \mathrm{~mm} \mathrm{Hg}$ ) or hypertension, increasing to $78 \%$ above age 60 , indicating significant cardiovascular risk in this cohort. Longitudinal analyses of blood pressure reveal important heterogeneity. For women aged 45 to 74 years (but not for men) or men and women aged $75+$, there is an increase in systolic blood pressure of 2.5 base points during 2013 to 2017, corresponding to a 0.6 point increase of blood pressure per year (similar to the age gradient in Western, but more than that in some other low-income populations). ${ }^{15}$ The gender-difference potentially reflects

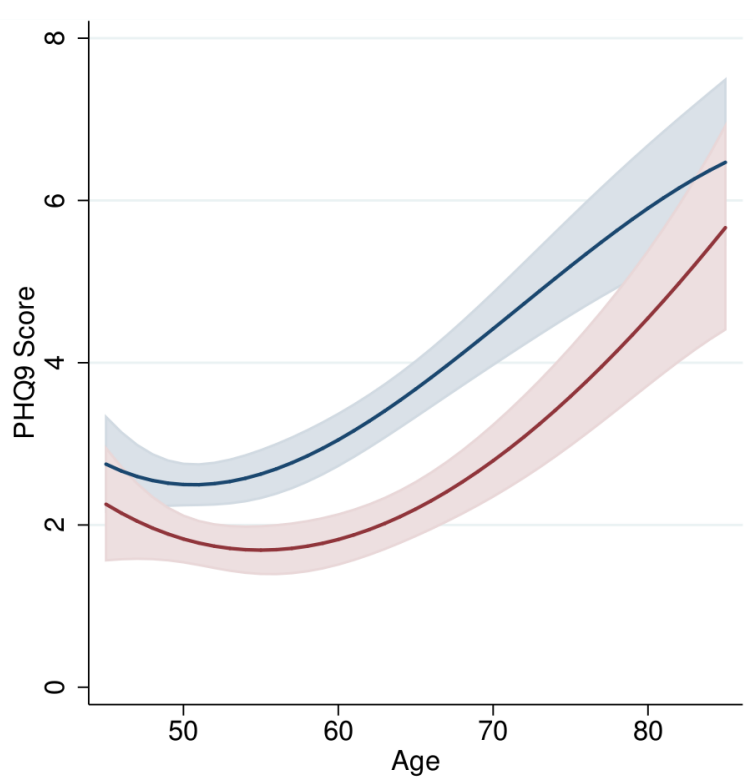

Figure 2 Age patterns of depression (PHQ-9 score) for mature adults, 2012 to 2013. Notes: PHQ-9 depression score by age for women and men. Figure shows marginal means (with 95\% Cls) obtained by regressing the respective mental health score on a cubic function of age, separately by sex. Analyses are pooled across 2012 and 2013 MLSFH-MAC surveys. Cls are adjusted for clustering within respondents. Analyses control for schooling, region and MLSFH-MAC wave. Religion is not controlled for because it is essentially collinear with region in the MLSFH-MAC; marital status is not controlled for because it is time-variant and endogenous with respect to mental health. MLSFH-MAC, Mature Adults Cohort of the Malawi Longitudinal Study of Families and Health; PHQ-9,9-item Patient Health Questionnaire.

the well documented sexual dimorphism of blood pressure changes with age. ${ }^{16}$ Important is also that, among MLSFH-MAC respondents who were measured with high blood pressure, only a small fraction had ever been diagnosed with hypertension by a healthcare professional, and an even smaller fraction was on treatment. ${ }^{17} 18$

\section{Subjective survival expectations and mortality}

In 2017, MLSFH-MAC respondents expected a hypothetical healthy individual to have a $70 \%$ chance of survival for the next 5 years, compared with $62 \%$ for someone who is HIV+, $49 \%$ for someone who is sick with AIDS and $57 \%$ for a HIV+ person who is treated with ART. Respondents reported median subjective probabilities for their own 5 -year survival of about $43 \%$ to $66 \%$, compared with $81 \%$ to $86 \%$ suggested by official life-tables (figure 5 ). This implies that rural Malawians underestimate their chances to survive 5 years by $33 \%$ on average. A 2017 MLSFH-MAC study documented that perceived population-level mortality risks-but not perceptions of own morality risks-are modifiable by providing objective life-table information on survival. Respondents who received the information subsequently had a $19 \%$ reduction in the propensity to have multiple sexual partners without using a condom, and an $8 \%$ increase in sexual abstinence; 


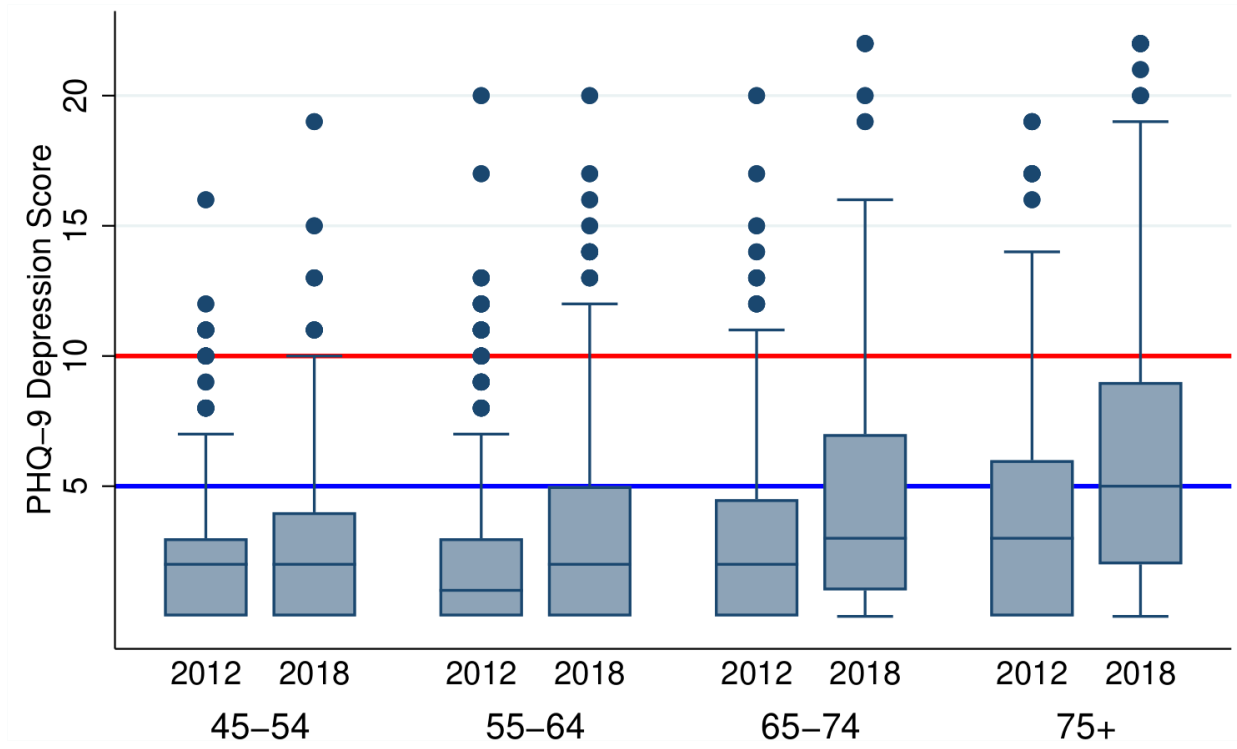

Figure 32012 to 2018 cohort change in PHQ-9 depression score, by age in 2018. Notes: Boxplots of PHQ-9 depression score in MLSFH-MAC cohort, by age in 2018. PHQ-9 scores are top-coded at a score of 22 (affecting very few observations). Analysis includes only respondents who participated in the 2012 and 2018 MLSFH-MAC surveys, and show the change in the distribution of PHQ-9 as the MLSFH-MAC cohort members aged during 2012 to 2018. Red line: PHQ-9 threshold for moderate depressive symptoms; blue line: PHQ-9 threshold for mild depressive symptoms. MLSFH-MAC,Mature Adults Cohort of the Malawi Longitudinal Study of Families and Health; PHQ-9,9-item Patient Health Questionnaire.

they also engaged in more forward-looking behaviours, for instance by increasing savings and investing more in agriculture. $^{19}$

\section{Overall relevance of findings}

The gains in adult life expectancy foreshadow a new challenge for which SSA LICs are ill prepared: the shifting disease burden in SSA towards chronic NCDs, which has been described as the new frontier in global health. ${ }^{20}$ The growing emphasis on NCDs in global health is due to at least two reasons: First, NCDs-among the MLSFH-MAC study participants and in SSA LIC populations more generally-are primarily CVD, hypertension and NCDsassociated functional disabilities. These NCDs increasingly contribute to disease burdens, thereby becoming leading causes of adult morbidity and mortality in SSA, while disease burdens attributable to communicable diseases

Table 5 HIV prevalence among 2017 MLSFH-MAC respondents, and HIV incidence among 2012 to 2017 MLSFH-MAC respondents

\begin{tabular}{|c|c|c|c|}
\hline & Women & Men & Total \\
\hline \multicolumn{4}{|l|}{2012 MLSFH mature adults $(n=1217)$} \\
\hline HIV prevalence, all 2012 respondents & $4.5 \%$ & $5.3 \%$ & $4.8 \%$ \\
\hline \multicolumn{4}{|l|}{ HIV prevalence among those who } \\
\hline Died by 2017 ( $n=125)$ & $6.8 \%$ & $7.6 \%$ & $7.2 \%$ \\
\hline \multicolumn{4}{|l|}{2017 MLSFH mature adults $(n=1555)$} \\
\hline HIV prevalence, all 2017 respondents & $8.8 \%$ & $7.6 \%$ & $8.3 \%$ \\
\hline HIV prevalence, 2017 respondents aged 45 to 49 & $14.6 \%$ & $9.2 \%$ & $12.6 \%$ \\
\hline HIV incidence (\%, among respondents tested & $0.15 \%$ & $0.83 \%$ & $0.43 \%$ \\
\hline \multicolumn{4}{|l|}{ both 2012 and 2017) } \\
\hline
\end{tabular}

1200 MLSFH-MAC respondents were tested for HIV in 2012, updating earlier HIV tests from 2004 to 2008 available through linkage to MLSFH. 1551 MLSFH-MAC respondents were tested for HIV in 2017. Combined with earlier MLSFH HIV tests, HIV status information is available for 1247 MLSFH-MAC respondents in 2012, for 1598 in 2017 and for 1080 who were surveyed in both 2012 and 2017. MLSFH-MAC, Mature Adults Cohort of the Malawi Longitudinal Study of Families and Health. 
(A) Hand-grip strength 2012 \& 2017, same respondents, by baseline (2012) age group

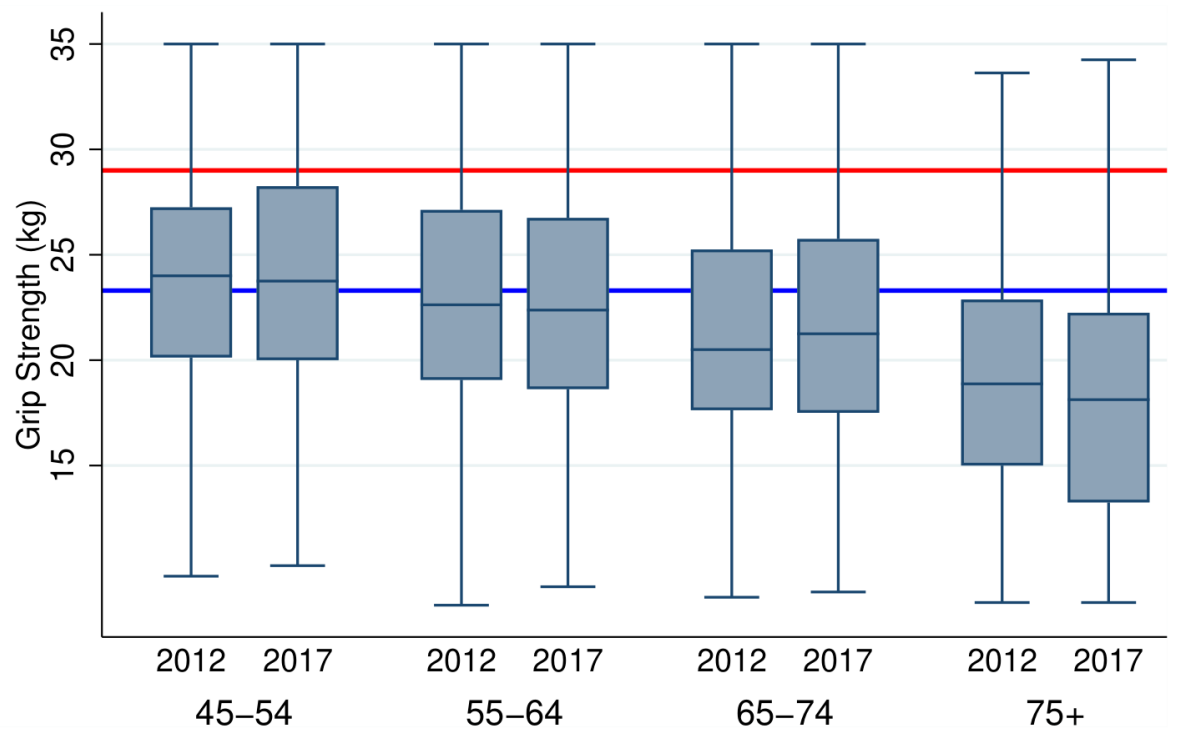

(B) Systolic blood pressure 2013 \& 2017, same respondents, by baseline (2012) age group

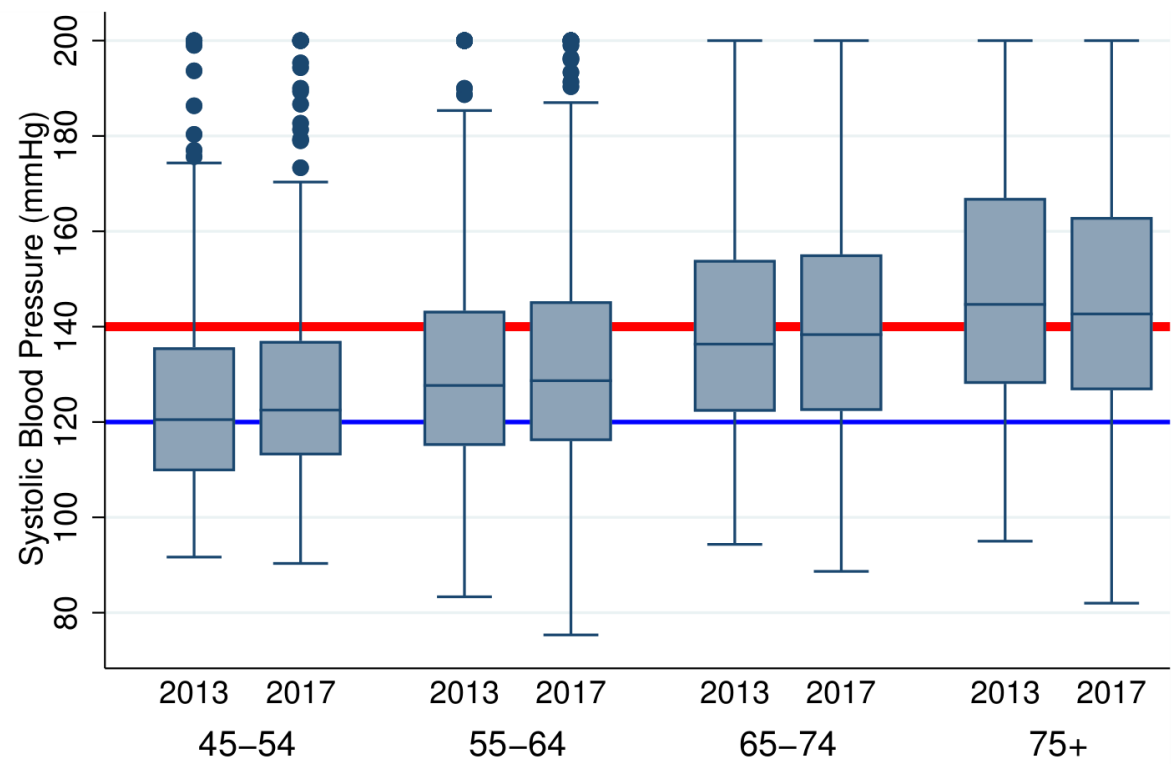

Figure 4 Notes: Boxplots of grip strength (Panel A) and systolic blood pressure (Panel B) in the MLSFH-MAC cohort, by age in 2017. Both genders combined. Analyses include only respondents who participated in the 2012 (2013) and $2017 \mathrm{MLSFH}$ MAC surveys (=surveys with the initial and most recent measures). Additional notes for top panel: Grip strength is measured in $\mathrm{kg}$, combining average left and right hand, each based on 3 measurements. Red line indicates median grip strength for the US population age 55 to 64 years old estimated from the Health and Retirement Study (HRS); blue line indicates 25th percentile of grip strength for the HRS population age 55 to 64 years. Additional notes for bottom panel: Systolic blood pressure is measured in mmHG, average value based on 3 measurements. Red line indicates $140 \mathrm{mmHG}$, a common cut-off for suggesting hypertension, and blue line indicates $120 \mathrm{mmHG}$, a common cut-off suggesting pre-hypertension.

have been decreasing. Second, NCDs and associated functional disabilities and physical health limitations that have been documented using the MLSFH-MAC have important limiting effects on adult economic activities, which in turn is likely to hamper benefits in SSA of a demographic dividend during which a growing population-share of economically-active adults provides. ${ }^{21}$ Scholars and NGOs have therefore argued that health research and policies in SSA LICs devote far too few resources to NCDs and their determinants/consequences. ${ }^{22}$ As a result, studies such as the MLSFH-MAC are critically important to fill very basic knowledge gaps that continue to exist with respect to the prevalence of NCDs, their risk factors and the driving forces behind their relatively recent dramatic increase in SSA LICs. The current knowledge is also very inadequate for understanding the consequences of these shifting disease burdens, and for developing adequate public health policy and health-system responses in SSA LICs, including those highlighted in the Malawi National Health Sector Strategic Plan. Such context-specific 


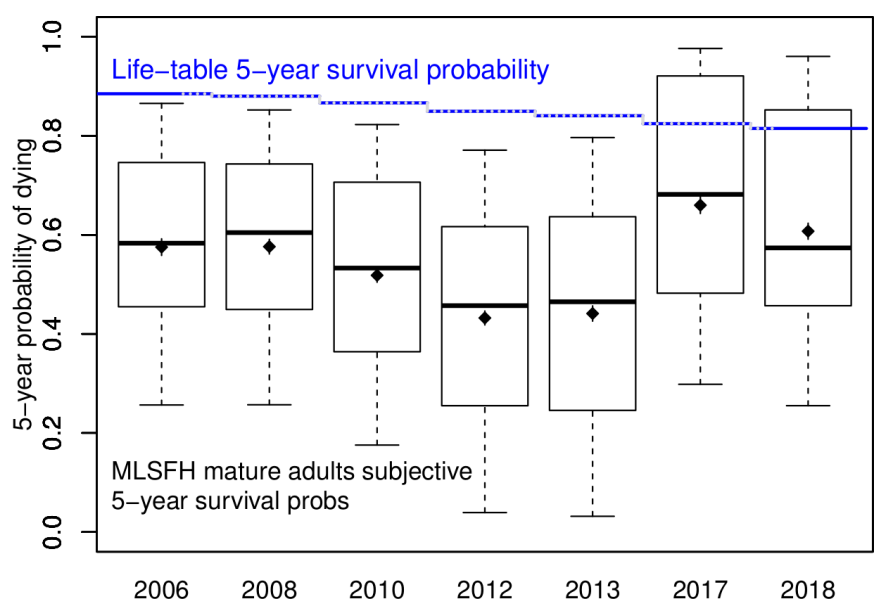

Figure 5 Subjective 5-year survival probabilities for MLSFHMAC respondents, 2006 to 2018. Notes: the boxplot-like graph displays the mean (dot) and median (centre line) of the corresponding 5-year survival expectations, as well as the $10^{\text {th }}$ (lower whisker), $25^{\text {th }}$ (bottom of box), $75^{\text {th }}$ (top of box) and $90^{\text {th }}$ (upper whisker) percentiles of the distribution. Lifetable survival probabilities are merged by age and gender with the UN Malawi 2005 to 2015 lifetables, ${ }^{23}$ and combined into an average to match the age-distribution and genderdistribution of the MLSFH-MAC cohort. MLSFH-MAC,Mature Adults Cohort of the MalawiLongitudinal Study of Families and Health.

evidence is necessary as findings from more developed contexts are generally not sufficient for addressing the distinctive knowledge gaps about NCDs in SSA LICs as epidemiological, market, policy and resource contexts differ so much that useful guidance cannot simply be transferred from high-income country research studies.

\section{STRENGTHS AND LIMITATIONS}

The MLSFH-MAC fills an important research need by providing two decades of cohort data on social, economic and health aspects of ageing, covering a period in which cohort members also faced the HIV/AIDS epidemic that further heightened the adversities resulting from poverty and a high burden of other diseases. Cohort data are wideranging, including NCDs, physical and mental health, cognition, social networks, sexual behaviour, social and economic conditions, household structure, risk perceptions and other aspects. Complemented by contextual and qualitative information, the extensive MLSFH-MAC data facilitate a life-course perspective on ageing that reflects the dynamic and distinct settings in which people reach older ages in SSA LICs. Across many domains, the MLSFH-MAC also allows for comparative research with global ageing studies through harmonised measures and instruments.

The MLSFH-MAC also compensates for attrition by enrolling new cohort members on reaching age 45, and the data reflect the diversity of the rural Malawi population with its with diverse ethnic/religious composition and socioeconomic contexts. The cohort also includes an increasing peri-urban/urban subset of respondents who are retained as part of migration follow-ups. Other strengths of the MLSFH-MAC include the relatively large sample size, a high data quality resulting from the ability to build on the MLSFH experience and methodologies, the ability to link MLSFH-MAC cohort data between spouses and to prior life-course data dating back to 1998.

Some weaknesses of the MLSFH-MAC are noteworthy. The cohort is not a nationally representative sample, but instead represents mature adults in rural Malawi (specifically, rural areas in the three study districts). A limitation is also the relative lack of biomarker-based health data, which currently includes only HIV-testing and diabetes testing complemented by several measured health indicators. Another concern is related to attrition that is primarily due to mortality (table 2), while attrition from other reasons is relatively small given the extensive MLSFH-MAC efforts to locate respondents (including through migration follow-ups) and the strong ties to the MLSFH study communities that ensures high rates of study participation.

\section{COLLABORATION}

Public-use version of the 2012 (baseline) MLSFH-MAC data without identifying individual or village information can be requested on the project website at http:// www.malawi.pop.upenn.edu, and inclusion in the ICPSR (Inter-university Consortium for Political and Social Research) is pending. MLSFH-MAC data covering subsequent data collections will be made publicly available in the future. In the meantime, interested researchers can approach the MLSFH-MAC principal investigators (mailto:iliana@upenn.edu and/or mailto:hpkohler@ pop.upenn.edu) for sharing MLSFH-MAC data as part of collaborative research projects (if not overlapping with ongoing research projects, and subject to a Data Use Agreement).

\section{Twitter Collin F Payne @collinfpayne}

Acknowledgements In addition to the authors of this paper, members of the MLSFH-MAC study team who have made important contributions to the design and/ or implementation of the MLSFH-MAC during 2012 to 2018 include: Kathy Lowler, Michael Blank, Adeline Delavande, Victor Mwapasa, Jürgen Maurer, Andrew Zulu, Sophia Chae, Emanuel Szousa, Augustine Harawa, Thomas Anderson, Julio Romero Prieto, Alejandro Sanchez Bacerra, Dui Do, David Frankefield, Philip Anglewicz, Susan Watkins, Gifton Saizi, Blessings M'bwerazino, Martha Nkhukuzalira, Binny Kachepa, Amos Chikoti, Butsie Butao, Glyn Mgabie, Hastings Honde, Jacob Mkhwamba, Keston Mambo, Paul Chikwemba, Sydney Lungu, Wanangwa Nyirenda, Zaynab Mpaweni and Arthur Banda.

Contributors IVK and HPK conceptualised the MLSFH-MAC, designed the study instruments and implemented the MLSFH-MAC data collections. IVK wrote the first draft of the manuscript. IVK, HPK, CB, CFP, AC and FK analysed and interpreted the data. $\mathrm{CB}, \mathrm{JM} w$ and JMk contributed to the implementation of the study and development and testing of its instruments. All authors critically revised the manuscript and approved the final version.

Funding We gratefully acknowledge the generous support for the Mature Adults Cohort of the Malawi Longitudinal Study of Families and Health (MLSFH-MAC) by the Swiss Programme for Research on Global Issues for Development (SNF r4d Grant 400640_160374), National Institute on Aging (NIA R21 AG0537630), and pilot funding received through the Population Aging Research Center (PARC), 
supported by NIA P30 AG 012836, the Center for AIDS Research (CFAR), supported by NIAID Al 045008 and the Institute on Aging, all at the University of Pennsylvania. We are also grateful for support for the Malawi Longitudinal Study of Families and Health (MLSFH) by the Rockefeller Foundation, the National Institute of Child Health and Human Development (NICHD R03 HD058976, R21 HD050653, R01 HD044228, R01 HD053781, R01 HD087391), the Boettner Center for Pensions and Retirement Security and the NICHD Population Research Infrastructure Program (R24 HD044964).

Map disclaimer The depiction of boundaries on this map does not imply the expression of any opinion whatsoever on the part of BMJ (or any member of its group) concerning the legal status of any country, territory, jurisdiction or area or of its authorities. This map is provided without any warranty of any kind, either express or implied.

Competing interests None declared.

Patient and public involvement Patients and/or the public were involved in the design, or conduct, or reporting, or dissemination plans of this research. Refer to the Methods section for further details.

Patient consent for publication Not required.

Ethics approval The data collections of the MLSFH-MAC and MLSFH have been approved by the IRB Board at the University of Pennsylvania (IRB Protocols \#815 016 and \#826828), and in Malawi, the MLSFH-MAC and MLSFH research has been approved by the Ethics Committee of the College of Medicine, Malawi (COMREC, Protocols \#P01/12/1165 and \#P.04/17/2160) and the National Health Sciences Research Committee (NHSRC, Protocol \#19/01/2214).

Provenance and peer review Not commissioned; externally peer reviewed.

Data availability statement Data are available upon reasonable request. Publicuse version of the 2012 (baseline) MLSFH-MAC data without identifying individual or village information can be requested on the project website at http://www. malawi.pop.upenn.edu, and inclusion in the ICPSR is pending. MLSFH-MAC data covering subsequent data collections will be made publicly available in the future. In the meantime, interested researchers can approach the MLSFH-MAC principal investigators (mailto:iliana@upenn.edu and/or mailto:hpkohler@pop.upenn.edu) for sharing MLSFH-MAC data as part of collaborative research projects (if not overlapping with ongoing research projects, and subject to a Data Use Agreement).

Open access This is an open access article distributed in accordance with the Creative Commons Attribution Non Commercial (CC BY-NC 4.0) license, which permits others to distribute, remix, adapt, build upon this work non-commercially, and license their derivative works on different terms, provided the original work is properly cited, appropriate credit is given, any changes made indicated, and the use is non-commercial. See: http://creativecommons.org/licenses/by-nc/4.0/.

Author note Subject Headings: Older Adults, Health, HIV/AIDS, Health Behavior, Intergenerational Relations, Family and Household, Family Demography, Population Dynamics. Mental health, cognition, depression, sub-Saharan Africa, Malawi.

ORCID iD

lliana V Kohler http://orcid.org/0000-0001-5114-546X

\section{REFERENCES}

1 WHO. Global action plan for the prevention and control of NCDS 2013-2020. Geneva, Switzerland: World Health Organization (WHO), 2013. http://www.who.int/nmh/events/ncd action plan

2 Kohler H-P, Watkins SC, Behrman JR, et al. Cohort profile: the Malawi longitudinal study of families and health (MLSFH). Int J Epidemiol 2015;44:394-404.

3 Sonnega A, Faul JD, Ofstedal MB, et al. Cohort profile: the health and retirement study (HRS). Int J Epidemiol 2014;43:576-85.
4 Kohler HP, Ciancio A, Kohler IV, et al. The benefits of knowledge: short-term effects of providing information about mortality risks to mature adults in Malawi. Poster presented at the 2018 Annual Meeting of the Population Association of America, Denver, CO, 2018.

5 Ohrnberger J, Anselmi L, Fichera E, et al. Validation of the SF12 mental and physical health measure for the population from a lowincome country in sub-Saharan Africa. Health Qual Life Outcomes 2020;18:78

6 Kohler IV, Payne CF, Bandawe C, et al. The demography of mental health among mature adults in a low-income, High-HIV-Prevalence context. Demography 2017;54:1529-58.

7 Myroniuk T, Kohler HP, Kohler IV. Marital Dissolutions and changes in mental health: evidence from rural Malawi. University of Pennsylvania, Population Center (PSC/PARC) Working Paper 202032, 2020.

8 Payne CF, Kohler IV, Bandawe C, et al. Cognition, health, and well-being in a rural sub-Saharan African population. Eur J Popul 2018;34:637-62.

9 Payne CF, Kohler H-P. The population-level impact of public-sector antiretroviral therapy rollout on adult mortality in rural Malawi. Demogr Res 2017;36:1081-108.

10 Baranov V, Kohler H-P. The impact of AIDS treatment on savings and human capital investment in Malawi. Am Econ J Appl Econ 2018;10:266-306.

11 Harries AD, Ford N, Jahn A, et al. Act local, think global: how the Malawi experience of scaling up antiretroviral treatment has informed global policy. BMC Public Health 2016;16:938.

12 Kämpfen F, Kohler IV, Bountogo M, et al. Using grip strength to compute physical health-adjusted old age dependency ratios. SSM Popul Health 2020;11:100579.

13 Kuh D, Hardy R, Butterworth S, et al. Developmental origins of midlife grip strength: findings from a birth cohort study. J Gerontol $A$ Biol Sci Med Sci 2006;61:702-6.

14 Sayer AA, Syddall H, Martin H, et al. The developmental origins of sarcopenia. J Nutr Health Aging 2008;12:427-32.

15 Gurven M, Blackwell AD, Rodríguez DE, et al. Does blood pressure Inevitably rise with age? Hypertension 2012;60:25-33.

16 Chobanian AV, Bakris GL, Black HR, et al. Seventh report of the joint National Committee on prevention, detection, evaluation, and treatment of high blood pressure. Hypertension 2003;42:1206-52.

17 Kohler IV SN, Bandawe C, Kohler HP. Hypertension without conventional risk Factors-High prevalence of high blood pressure in a low risk, low income African population. Paper presented at the 2018 Annual Meeting of the Population Association of America, Denver, CO, 2018.

18 Ciancio A, Kämpfen F, Kohler H-P, et al. Health screening for emerging disease burdens among the global poor: Evidence from Sub-Saharan Africa, forthcoming in the Journal of Health Economics. University of Pennsylvania Population Center (PSC/PARC) Working Paper 2020-41, 2020.

19 Ciancio A, Delavande A, Kohler HP, et al. Mortality risk information, survival expectations and sexual behaviors. University of Pennsylvania Population Center (PSC/PARC) Working Paper 202039, 2020.

20 Greenberg H, Raymond SU, Leeder SR. The prevention of global chronic disease: academic public health's new frontier. Am J Public Health 2011;101:1386-90.

21 Behrman JR, Kohler H-P. Population Quantity, Quality, and Mobility.. In: Allen F, Behrman JR, Birdsall N, et al, eds. Towards a better global economy: policy implications for global citizens in the 21st century. Oxford, UK: Oxford University Press, 2014.

22 Beard J, Biggs S, Bloom D, et al. Global population ageing: peril or promise?. PGDA working paper No. 89 (global agenda Council on ageing Society), 2012. Available: http://www.hsph.harvard.edu/pgda/ working.htm

23 UN Population Division. World population prospects, the 2017 revision: key findings; 2017 . United nations, department of economic and social Affairs, population division. Available: http://esa.un.org/ unpd/wpp/ 